\title{
MODELING OF CLAMPING MAGNETS INTERACTION WITH FERROMAGNETIC SURFACE FOR WHEEL MOBILE ROBOTS
}

\author{
Yuriy Kondratenko 1), Yuriy Zaporozhets ${ }^{1)}$, Joachim Rudolph 2), \\ Oleksandr Gerasin ${ }^{3)}$, Andriy Topalov ${ }^{3)}$, Oleksiy Kozlov ${ }^{3)}$ \\ 1) Petro Mohyla Black Sea National University, 10 68th Desantnykiv st., Mykolaiv, 54003, Ukraine \\ y_kondrat2002@yahoo.com, yuriy.kondratenko@chmnu.edu.ua,umz-286@bigmir.net \\ 2) Saarland University, Campus A5 1, Saarbrucken, 66123, Germany, j.rudolph@1sr.uni-saarland.de \\ 3) Admiral Makarov National University of Shipbuilding, 9 Heroes of Ukraine ave., Mykolaiv, 54025, Ukraine \\ oleksandr.gerasin@nuos.edu.ua,topalov_ua@ukr.net,kozlov_ov@ukr.net
}

Paper history:

Received 11 January 2018

Received in revised form 03 March 2018

Accepted 15 March 2018

Available online 31 March 2018

Keywords:

mobile robot;

magnetically operated mover;

electromagnetic field;

clamping electromagnet;

clamping force control system;

finite element method;

method of secondary sources.

\begin{abstract}
The mathematical models of clamping permanent and electromagnets for clamping force determination by means of field theory (method of secondary sources and features of integrated forces calculation) are offered for magnetically operated movers of mobile robots (MR) for moving on inclined or vertical ferromagnetic surfaces. The results of experimental investigations of magnetic induction and clamping force measurements in different spatial positions of clamping magnet relative to the ferromagnetic surface are given. The developed simulation models based on field theory and finite element method allow to validate the obtained functional dependencies with respect to gaps between magnet and working surface, caused by excrescences of shells, and ferromagnetic surface thickness changes due to its technological features, as well as help at the stage of MR's design. Authors propose control and measuring system of clamping force that can be successfully used for new and existing MRs' constructions for their reliable operating on complex surfaces taking into account the nature of their uncertainties (ferromagnetic or non-ferromagnetic).
\end{abstract}

Copyright $@$ Research Institute for Intelligent Computer Systems, 2018.

All rights reserved.

\section{INTRODUCTION}

The mobile robots, able to move on an inclined surface by means of different types of clamping devices and appropriate movers, are widely used for automation of industrial technological operations in the developed countries [1-4]. Such MRs allow to reduce performance time and risks for human health and life at stripping of large complex surfaces (in ship building and ship repair), deactivation in radiation conditions, firefighting, dyeing, inspection, welding, etc. according to [5-7]. So, any automatic control system (ACS) of such robots must provide reliable adhesion of MR's construction to the different working surfaces without slipping from a specified location [2].

Magnetically operated clamping devices of MRs are built on the basis of clamping electromagnets (CE), which provide reliable adhesion to the ferromagnetic working surfaces and contribute to improve reliability and productivity of the different technological operations performance $[8,9]$. Also, the clamping force control leads to reliability and energy efficiency improving of the MR's moving process by traction elements or separate movers.

Some cases of the most wide-spread MR's CEs and traction or fastening elements position near working surface (for example, parts of ship hull) are shown in Fig. 1. The air gap between clamping element and surface is denoted as $\delta$ and $s$ is a thickness of a ferromagnetic surface (FS).

The first case (Case A in Fig. 1) of CE and FS placement is usually met at the ship hull assembling. Steel plates are clean and smooth in this case. Almost all of CE clamping surface presses evenly to FS $(\delta \approx 0)$.

The second case deals with maintenance operations such as ship cleaning and repairing (Case B in Fig. 1). FS is covered with sea shells and seaweed. Such coating significantly decreases the 
ship velocity characteristics and must be periodically removed by specialized cleaning machines, in particular, mounted on MR's transport platform. CE may meet different non-ferromagnetic obstacles on the way to FS in this case $(\delta \neq 0, \delta=v a r)$. Moreover, the spatial position of $\mathrm{CE}$ depends on the nature of the working surface during MR movement: CE's clamping surface can be placed at angles to FS, which are usually changed for the new position.

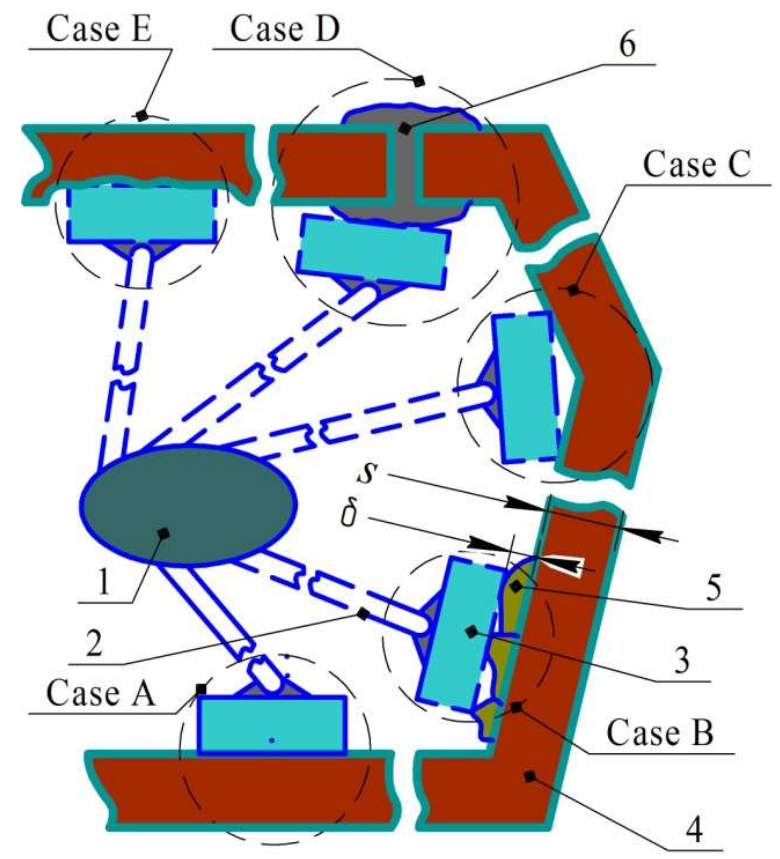

Fig. 1 - Some cases of MR's elements (1 - MR's transport platform; 2 - traction or fastening element; 3 - clamping electromagnet position on the working surface 4 with shells 5 and welded seam 6 .

MRs often have to operate in conditions of uncertainty [10] due to heterogeneity of the working surface like significant roughness, fouling or irregularities, for example, caused by human activity: empty holes, welded joints, construction elements, etc. Case $\mathrm{C}$ in Fig. 1 is similar to the previous but the air gap changes are caused by hull construction features. Case D in Fig. 1 shows welded seam presence on FS. Now the FS thickness changes $(s=$ var), it increases. In shipbulding, in particular, ship repairing, Case E is more commonly used, because every day ship hulls corrode and deteriorate during functioning in marine and fresh water. So, their thickness decreases during operation. As a result of large ammount of previous repairs, FS's thickness is not the same in almost the entire hull square $(s=$ var).

According to the above-mentioned it may be seen that non-ferromagnetic and ferromagnetic features of working surfaces with respect to the field interaction and clamping force generation can be inspected by consideration of general cases (Case A - Case E in
Fig. 1). MR's ACS should consider all situations of CE positioning.

The clamping force ACS cannot receive the feedback information about the current value of clamping force, generated by CE [11], when MR carries out given technological operations.

Direct measurements by individual strain gauges (Fig. 2, a [12]) are not appropriate for MR's CE, because the surface is uncertain (as can be seen in Fig. 1 a point of clamping force applying is unknown). Some materials according to their electrical conductivity may be applicated as a force sensor (Fig. 2, b) [13, 14]. They can cover the CE clamping surface, but their using deals with significant clamping force losses and makes it almost impossible such sensor's calibration procedure. Slip displacement sensors application (Fig. 2, c) [14-16] for clamping force measurements is not appropriate also, because $\mathrm{CE}$ can take such position when under maximum control signal, it isn't able to create a required clamping force value (in fact, emergency situation).

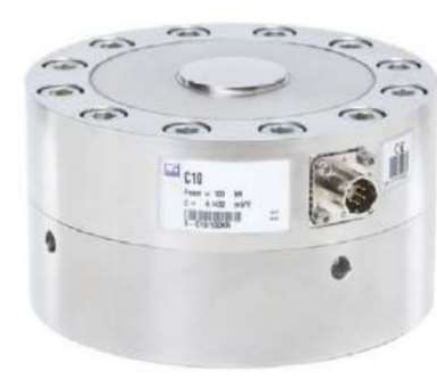

a

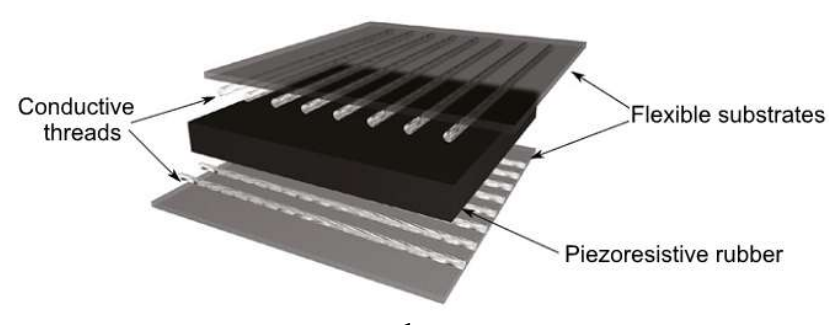

b

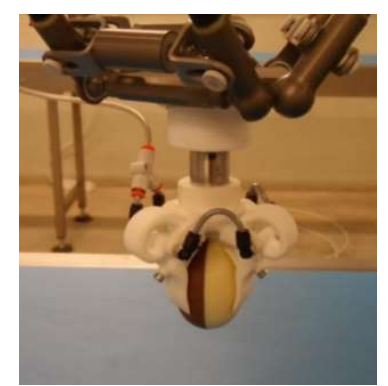

C
Fig. 2 - Widely used tools for clamping force measurements: a - force gauge; $b$-device based on electrically conductive elastomers; $\mathbf{c}$ - robot's gripper with built-in slip displacement sensors.

Therefore, in these conditions the indirect estimation can be used as the most suitable way of the clamping force determination [17, 18]. This process comprises the unknown system output evaluation by known system input with necessary physical quantities measuring. Besides considering the above, there is an urgent task of choosing CE number and their power at the MR designing stage. But it is a very difficult and multiparametric problem that requires a large number of calculations based on the field theory methods $[18,19]$. At the 
same time, computer modeling methods [20, 21] show their effectiveness and inexpensiveness, comparing with other approaches, especially experimental. So, at the stage of MR's development it is advisable to consider computer simulation opportunities to study the behavior of electromagnetic objects and CEs effectiveness.

Thus, it is reasonable to explore the $\mathrm{CE}$ model and to simulate the field interaction features of CE with working surface due to essential dependence of clamping force on the non-ferromagnetic gap, for example, defined by excrescences of shells, and also from other uncertainties of the working surface (ferromagnetic surface thickness changes caused by its technological features) and external factors of the environment, which don't depend on the MR operator and cannot be accurately measured or calculated by the main ACS of MR.

Therefore, the aim of this work is development and research of the mathematical model of the CE that is used in wheeled mobile robots on the basis of the field theory approaches and equations, as well as their solving by finite element method and soft computing techniques.

\section{MOBILE ROBOT WITH MAGNETICALLY OPERATED WHEEL- MOVERS}

MR, equipped with magnetically operated wheelmovers [18], is shown in Fig. 3. All wheel-movers are fixed on the transport platform 1 with technological equipment or cargo 10. On-board multichannel control unit includes the clamping force ACS (CFACS) that provides a required value of each CE's clamping force for reliable keeping of MR on working surface. This system forms CEs' supplying voltages for each magnet separately. Geometrical axis of such wheel-mover 2 matches with the point of intersection bisectors of wheel polygon interior angles. Rigid spokes 3 with split sections 4 and hub 5 provide structural strength of the wheel-mover construction [18].

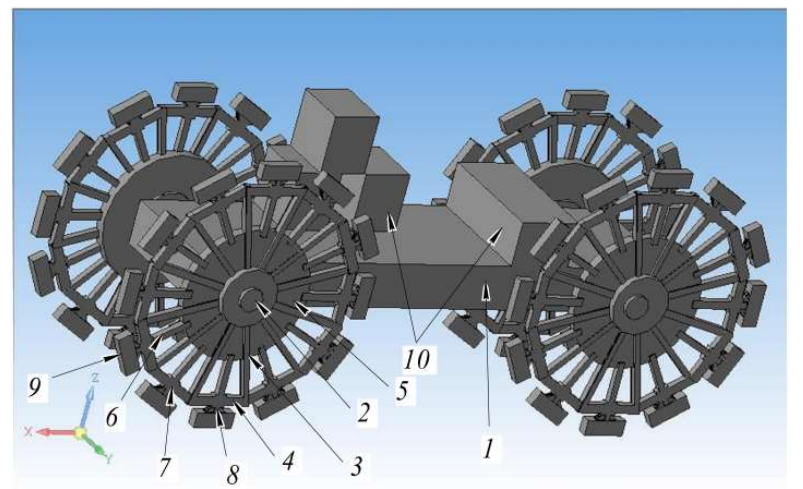

Fig. 3 - MR's construction with magnetically-operated wheel-movers.
Support-traction units perform MR's movement on working surface [11]. Each such unit includes sliding spokes with mounted in the hub 5 fixed elements and moving rods 6 , which are put forward to machined ferromagnetic surface through the guide bushings 7 in split sections 4. Clamping electromagnets 9 are attached by hinges 8 to the free ends of the rods 6 and provide the given value of clamping force. With straightforward movement all magnetically operated wheel-movers are simultaneously operated in the same direction. The speeds of rotation and the translational movement of the corresponding movers are adjusted in case of rotation of the MR's body.

CEs of the MR can be additionally equipped with permanent $\mathrm{NdFeB}$ magnets. Addition of permanent magnets to the clamping device improves the reliability of the robot's movement and technological operations performing on complex ferromagnetic surfaces in the event of a power system's malfunction. Also, MR can be equipped with the following processing equipment: hydroabrasive cleaning system, welding apparatus, paint spraying machine, etc.

MR is held on the working surface by clamping force formation system that is the part of the overall MR's ACS [18]. There are controlled convertor, CE, force regulator and a set of sensors in the system. The value of current clamping force can be determined by force identifier [18] using other measurements from sensors. So, let's consider electromagnetic processes of $\mathrm{CE}$ interaction with ferromagnetic surface for investigations of the most significant factors in clamping force forming as well as ways of their further consideration at force identifier development.

\section{CLAMPING MAGNETS' DESCRIPTION BY THE FIELD THEORY}

One or more clamping magnets (CMs) can be used to provide the required clamping force in MR's designs. Herewith, at the development stage for the proposed design of the propulsion wheel-mover several basic positions should be defined:

- the required number of $\mathrm{CMs}$ with the appropriate parameters for creation of the required magnetic force to hold the MR at manufacturing operations executing on surfaces with different slopes;

- the minimum number of CMs remaining in contact with the surface at performing rectilinear motion and maneuvering under the same conditions.

On the basis of the above it can be concluded that the task of power calculation of MR with $\mathrm{CMs}$ consists of determining of the normal and tangential forces applied to the working FS that are created by 
the CMs in an arbitrary spatial position relative to this surface.

The use of an electromagnet or a permanent magnet for MR adhesion to working surface imposes some features of magnetic induction and clamping force determination. So, let's consider a few methods of CMs' description (for permanent magnet and electromagnet).

\subsection{METHOD OF SECONDARY SOURCES FOR A PERMANENT CLAMPING MAGNET CALCULATION}

CMs based on permanent magnets are successfully used in constructions of cleaning and inspection MRs [4, 6-8]. The calculation scheme for solving the problem of determining of the clamping forces, applied to magnetic systems (Fig. 1), in a generalized form is shown in Fig. 4. The magnetic system of MR with $\mathrm{CM}$ consists of a permanent magnet (PM) and a FS, through which the magnetic flux of the PM is closed. The working PM 1 is shown in Fig. 4 in the position, where it is being detached from the FS by the driven wheel. An oblique gap is forming with a slope angle $\gamma$, which has the value $\delta$ over the center of the pole, $2-$ "mirror reflection" of the clamping magnet relative to the FS. The arrows indicate the direction of magnetization of PM. The segment $a b$ displays the pole edge of the magnet which, according to the direction of magnetization, is the "northern" $N$ pole, and the opposite edge is respectively the "southern" $S$ pole.

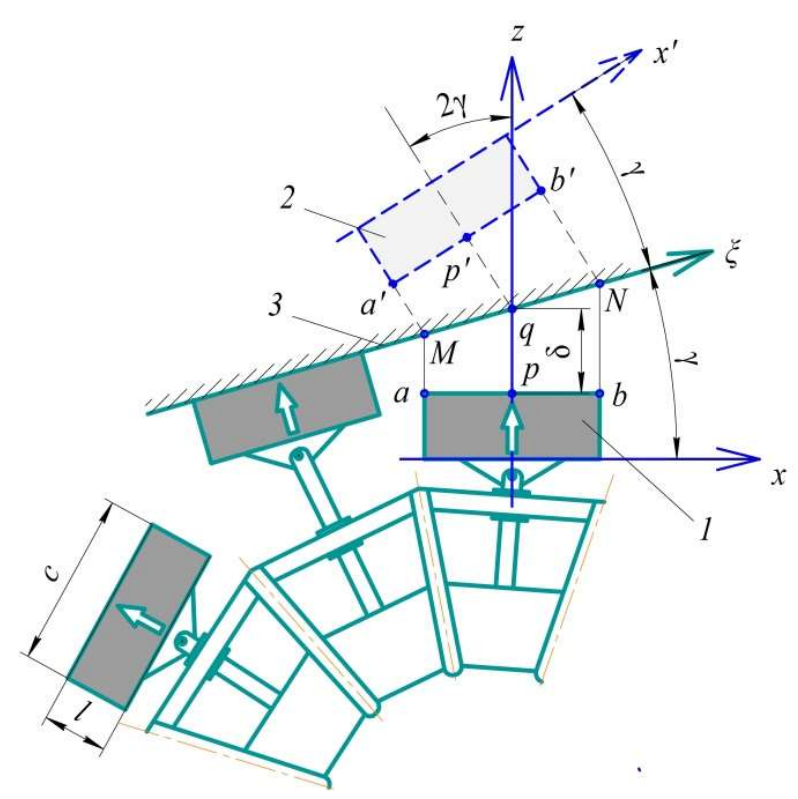

Fig. 4 -Calculation scheme of the magnetic system of MR with CM: $c$ and $l$ are width and height of the magnet pole (the size $d$ of the magnet along the $y$ axis, perpendicular to the plane of the figure, is not shown);

1 - working CM; 2 - "mirror reflection" of the CM relative to the $\mathrm{FS} ; 3$ - FS.
It is necessary to indicate generally accepted assumptions about the magnetic properties of the PM and FS to formulate the mathematical model of the system:

a) when modern highly coercive magnets are used in the device, their magnetization $J$ remains unchanged regardless of the position they occupy in the process of functioning relative to the FS and other magnets;

b) the magnetic permeability of the FS material is sufficiently large, i.e. we can do the assumption of its infinity $(\mu \rightarrow \infty)$.

Under such conditions the method of mirror reflections is usually used to solve problems of calculating of magnetic fields and the forces created by $\mathrm{CM}$ and FS. The simulation model of the CM is used in the form of a simple layer of magnetic charges, that are uniformly distributed at the poles of the magnet with a density $\sigma_{\mathrm{M}}= \pm \mu_{0} J$, for the power calculation of the MR with CM. The adequacy of such model is shown in [22]. It can be also used for the calculations of vertical $H_{z}$ as well as transverse $H_{x}$ and $H_{y}$, relative to the magnet, components of the magnetic field at an arbitrary point in the space $P$ with relations of the form

$$
\mathbf{H}(P)=-\frac{J}{4 \pi} \int_{S_{N}+S_{S}} \operatorname{grad}_{P}\left(\frac{1}{R_{\mathrm{PM}}}\right) d s,
$$

where $R_{P M}$ is the radius-vector from the point of integration $M$ to the point $P$, and

$$
R_{\mathrm{PM}}=\sqrt{\Delta x_{P M}^{2}+\Delta y_{P M}^{2}+\Delta z_{P M}^{2}} .
$$

The performance of these integro-differential operations over the area of the pole edges $S_{N}$ and $S_{S}$ of magnets makes it possible to obtain finite formulas for calculating of the field components [15]:

$$
\begin{aligned}
& H_{z}(P)=-\frac{J}{4 \pi} \sum_{i, j, k=1}^{2}(-1)^{i+j} \operatorname{arctg}\left(\Delta x_{i} \Delta y_{j} /\right. \\
& \left./(-1)^{k} \Delta z_{k} \sqrt{\Delta x_{i}^{2}+\Delta y_{j}^{2}+\Delta z_{k}^{2}}\right),
\end{aligned}
$$

where $\Delta x_{i}=x_{P}+(-1)^{i} c / 2, z_{1}=l, z_{2}=0$, $\Delta y_{j}=y_{P}+(-1)^{j} d / 2, \quad \Delta z_{k}=z_{k}-z_{P} \quad$ in the coordinate system (see Fig. 4),

$$
\begin{aligned}
& H_{x}(P)=\frac{J}{4 \pi} \sum_{i, j, k=1}^{2}(-1)^{i+j} \operatorname{Arsh}\left(\Delta y_{j} /\right. \\
& \left./(-1)^{k} \sqrt{\Delta x_{i}^{2}+\Delta z_{k}^{2}}\right)
\end{aligned}
$$




$$
\begin{aligned}
& H_{y}(P)=\frac{J}{4 \pi} \sum_{i, j, k=1}^{2}(-1)^{i+j} \operatorname{Arsh}\left(\Delta x_{i} /\right. \\
& \left./(-1)^{k} \sqrt{\Delta y_{j}^{2}+\Delta z_{k}^{2}}\right) .
\end{aligned}
$$

Now, according to the chosen model, the effort created by the magnet 1 relative to the FS 3 (see Fig. 4) can be defined as the Coulomb interaction between the magnetic field of the $\mathrm{CM}$ and the magnetic charges located at the poles of the "mirrored" magnet 2 [15]. Each component of the field $H_{\lambda}$ (coordinate index $\lambda$ takes the values $x, y$ or $z$ in the three-dimensional coordinate system) creates the relevant part of the force, distributed over the entire area of the verge pole $a^{\prime} b^{\prime}$ with the density:

$$
f_{\lambda}\left(x_{k}^{\prime}, y_{k}^{\prime}\right)=\mu_{0} J H_{\lambda}\left(P_{k}\right)=\mu_{0} J H_{\lambda}\left(x_{k}^{\prime}, y_{k}^{\prime}\right) \text {, }
$$

where the point $P_{k}=P\left(x_{k}^{\prime}, y_{k}^{\prime}\right)$ is the center of the $k$-th elementary area of the reflected magnet flats $S_{N}^{\prime}$ and $S^{\prime}: \Delta s^{\prime}{ }_{k}=\Delta x_{k}{ }_{k} \Delta y^{\prime}{ }_{k}$. The coordinates of the points $P\left(x_{k}^{\prime}, y_{k}^{\prime}\right)$ are calculated according to the geometric parameters (see Fig. 4).

By making numerical integration of the density of efforts (4) in the area of the pole edge, that is actually summing up each component on all sites, we find components of the main vector of the force, which generally acts on the pole edge $a^{\prime} b^{\prime}$ :

$$
F_{\lambda}=\int_{a^{\prime}}^{b^{\prime}} f_{\lambda}\left(x^{\prime}, y^{\prime}\right) d x^{\prime} d y^{\prime} \cong \sum_{k} f_{\lambda}\left(x_{k}^{\prime}, y_{k}^{\prime}\right) \Delta s_{k}^{\prime} .
$$

Next, using (6) and (7) we find the coordinates of the centers of parallel forces:

$$
\begin{aligned}
& x_{C, \lambda}^{\prime}=\sum_{k} x_{k}^{\prime} f_{\lambda}\left(x_{k}^{\prime}, y_{k}^{\prime}\right) \Delta s_{k}^{\prime} / F_{\lambda} ; \\
& y_{C, \lambda}^{\prime}=\sum_{k} y_{k}^{\prime} f_{\lambda}\left(x_{k}^{\prime}, y_{k}^{\prime}\right) \Delta s_{k}^{\prime} / F_{\lambda} ; \\
& z_{C, \lambda}^{\prime}=\sum_{k} z_{k}^{\prime} f_{\lambda}\left(x_{k}^{\prime}, y_{k}^{\prime}\right) \Delta s_{k}^{\prime} / F_{\lambda} .
\end{aligned}
$$

Finally, for the system (5) of the three forces $F_{\lambda}$ ( $\lambda$ takes the values 1,2, 3 which correspond to the projections on the $\mathrm{x}, y$ or $z$ axis) with the application points $C_{\lambda}$, whose coordinates are determined by $(8)-$ (10), we obtain the resultant vector and the resultant moment of the forces:

$$
\begin{gathered}
\bar{F}_{M}=\sum_{\lambda=1}^{3} \bar{F}_{\lambda}=\bar{e}_{x} F_{x}+\bar{e}_{y} F_{y}+\bar{e}_{z} F_{z} ; \\
\bar{M}_{Q}=\sum_{\lambda=1}^{3} \bar{r}_{Q, C_{\lambda}} \times \bar{F}_{\lambda},
\end{gathered}
$$

where $\bar{r}_{Q, C_{\lambda}}$ is the radius-vector, taken from the concentration center of the system of forces $Q$ to the point $C_{\lambda}$ of application of the force $F_{\lambda}$.

\subsection{PECULIARITIES OF INTEGRATED FORCES CALCULATION FOR CLAMPING ELECTROMAGNETS}

The use of an electromagnet for MR's adhesion to working FS imposes some features of magnetic induction and clamping force determination.

Phenomenological basis of the functioning of the magnetically operated MR wheel-mover, which is considered in [18], is forming of the clamping force (CF) of CE due to magnetic interaction (with the help of magnetic field $\hat{\mathbf{H}}$ ) of FS elements with the poles of an electromagnet. Each component of the field $H_{\lambda}$ (coordinate index $\lambda$ takes the values $x, y$ or $z$ in the three-dimensional coordinate system) creates the relevant part of the force, distributed over the entire area of the edge pole with the density:

$$
f_{\lambda}\left(x_{k}, y_{k}\right)=\sigma_{\mathrm{M}}\left(x_{k}, y_{k}\right) H_{\lambda}\left(x_{k}, y_{k}\right) \text {, }
$$

where the point $\left(x_{k}, y_{k}\right)$ is the center of the $k$-th elementary area of the magnet pole; $\sigma_{\mathrm{M}}$ is the density of magnetic charges and $H_{\lambda}$ are the relevant components of the magnetic field at points $\left(x_{k}, y_{k}\right)$.

Comparing (6) and (13) equations, it is clear whether a permanent CM describing its magnetization remains (is) the same over its surface in accordance with the accepted model of the CM or changes. At the same time for CE the density of "magnetic charges" $\sigma_{M}$ is a function of the pole edge coordinates $\left(x_{k}, y_{k}\right)$ that is determined by the solution of the field problem.

Therefore, the CEs are equipped with Hall sensors for measuring induction values and determining parameters of the magnetic flux and magnetic conductivity of the gap by means of calculations to track the $\mathrm{CF}$ variations. For this purpose the embedded programmed processor or logical controller is added as a part of the force observer [18], which implements the algorithm for computing of magnetic flux and magnetic conductivity based on the solution of the Laplace equation for the potential of a magnetic field in a specific form:

$$
\begin{aligned}
& \Delta U(x, y, z) \equiv \frac{\partial^{2} U}{\partial x^{2}}+\frac{\partial^{2} U}{\partial y^{2}}+\frac{\partial^{2} U}{\partial z^{2}}=0 \Rightarrow \\
& \Rightarrow \partial^{2} U / \partial x^{2}+\partial^{2} U / \partial y^{2}=-\partial^{2} U / \partial z^{2} \equiv-\rho_{M},
\end{aligned}
$$


which can be interpreted as a Poisson equation in two-dimensional rectangular area (the plane of electromagnet pole), where $U$ - magnetic potential, $\rho_{\mathrm{M}}$ - density of equivalent "magnetic charge" that equals

$$
\rho_{\mathrm{M}}=\partial^{2} U / \partial z^{2} \equiv \partial B_{n} / \partial n
$$

where $\partial B_{n} / \partial n$ - normal to the surface of the pole derivative of the magnetic induction component.

By the method of Fourier two-dimensional potential has the following form:

$$
\begin{aligned}
& U(x, y)=\sum_{n=1}^{\infty}\left(A_{n} e^{\frac{n \pi}{b} x}+B_{n} e^{-\frac{n \pi}{b} x}\right) \sin \frac{n \pi}{b} y+ \\
& +\left(C_{n} e^{\frac{n \pi}{a} y}+D_{n} e^{-\frac{n \pi}{a} y}\right) \sin \frac{n \pi}{a} x,
\end{aligned}
$$

where $a$ and $b$-dimensions of rectangular pole; the unknown coefficients $A_{n}, B_{n}, C_{n}$ and $D_{n}$ are defined according to specific boundary conditions distinguishing this problem from traditional Dirichlet and Neumann problems, namely: for determination of $A_{n}, B_{n}, C_{n}$ and $D_{n}$ a system of linear algebraic equations is composed, in which derivatives of indicated potential $U(x, y)$ are equated to the measured values of induction, obtained by integrating of the expression $\rho_{\mathrm{M}}=-\Delta U(x, y)$. The algorithm of the specified procedure will be outlined in detail in another article.

The integrated value of the clamping force under uncertainty of the surface characteristics and unevenness of the gap between the CE and FS even with the same value of the CE's magnetic flux depends on the distribution of magnetic induction in the gap.

\section{MEANS FOR CLAMPING FORCE CONTROL SYSTEM IMPLEMENTATION}

As it can be seen in Section 3, magnetic induction measurements are required for determining clamping force. So, the authors proposed multichannel CFACS that includes sensor system (SS) based on Hall sensors, adders (A), regulators $(\mathrm{R})$, controlled keys $(\mathrm{CK})$, multichannel computing units (MCU) and reference voltage source (RVS). Let's consider one channel of the proposed system functioning in details.

Each CE is equipped with elastic insulating plate 4 that is fixed on the clamping surface of CE 1 and SS with at least five magnetic field sensors 3 (Fig. 5, a). One of the magnetic field sensors is mounted in the geometric center, and other sensors - in the peripheral area of the CE's clamping surface. The magnetic field sensors of each $\mathrm{CE}$ are placed between its clamping surface and elastic insulating plate of the corresponding CE. Ferromagnetic working surface is indicated by the number 2 (see Fig. 5, a).

The multichannel control unit is equipped with input/output groups, which quantity corresponds to the amount of wheel-mover's CEs. For one channel the outputs of the magnetic field sensors of $\mathrm{CE}$ are connected to the corresponding MCU, the output of which is connected to the second inverted input of the $\mathrm{A}$. The output of $\mathrm{A}$, in turn, is connected to the input of the clamping force $\mathrm{R}$, the output of which is connected to the corresponding input from the group of inputs of the control unit (Fig. 5, b). The first direct input of the $\mathrm{A}$ is connected through the corresponding controlled key with the output of the RVS (signal $U_{r}$ ). Also control input of the $\mathrm{CK}$ is connected to the corresponding output of the group of outputs of the multichannel control unit.
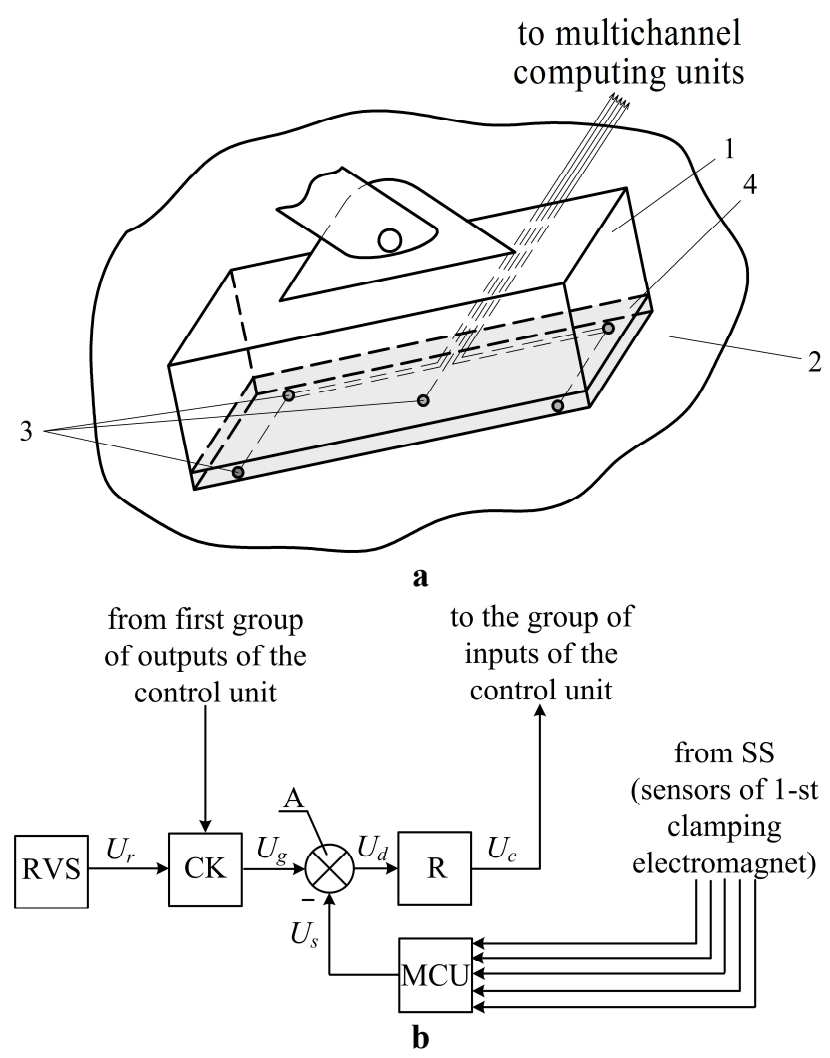

Fig. 5 - Scheme of CFACS: a - Hall sensors layout on CE's surface; $b$ - interaction between main parts.

MCU physically forms data signal $U_{s}$ that corresponds to the current value of the magnetic induction at the geometric center and in the peripheral area of the each CE's clamping surface (which is in the direct clutch with the ferromagnetic 
surface). Then, using specialized computing algorithm processing of the formed information signals is performed with the formation of the resulting signal $U_{s}$, which corresponds to the current value of clamping force of the corresponding $\mathrm{CE}$ in each current moment. And the resulting signal is compared with a set signal $U_{g}\left(U_{g}=U_{r}\right.$ or $U_{g}=0-$ depends of CK's current state) that corresponds to a set value of the clamping force.

Then the error signal $U_{d}$ between the set and the resulting signal is evaluated, whereby the value of the magnetic field induction of the corresponding CM changes until the full error compensation due to generating by $\mathrm{R}$ of control signal $U_{c}$ corresponding to error size (value). $\mathrm{R}$ can be built on the basis of methods of the classic control theory and also with artificial intelligent means [23].

\section{COMPUTER SIMULATION OF CLAMPING MAGNET'S INTERACTION WITH FERROMAGNETIC SURFACE}

It is known that numerical methods allow to find almost exact solution to the problem, because they are based on discrete calculation schemes [24, 25]. As a result of digitization, a continual system with countless degrees of freedom turns into a system with a finite number of degrees of freedom. The methods of finite elements, finite differences, finite volumes and others were the most developed [24].

As the studies in this paper show, the finite elements method is especially effective for the calculation of electromagnetic processes of machines and mechanisms. The idea of the finite elements method lies in the fact, that functional minimizing of variational problem is performed on the aggregate of functions, each of which is defined in its subarea [26]. The area, in which the differential equation solutions are searched for, is divided into finite number of subareas (elements). The form of approximating function is randomly selected in each element. In the simplest case it is a first-degree polynomial. Out of its element the approximating function is equal to zero. The functions values at the borders of the elements (nodes) provide solution to the problem and are beforehand unknown. The coefficients of approximating functions are commonly searched according to the conditions of values equality of neighboring functions on the borders between the elements. After that these coefficients are expressed through the functions values at the nodes of elements, and a system of linear equations is formed. Number of equations equals the number of unknown values in the nodes, in which the solution of the original system is searched for. It is also directly proportional to the number of items and only limited by the capabilities of computers [24].

As each of the elements is associated with a limited number of neighboring ones, the linear equations system has discharged appearance that greatly simplifies its solution. The use of systems of finite elements analysis makes it possible to study objects without creating their material prototypes, by forming and solving adequate mathematical models [25].

\subsection{PERMANENT CLAMPING MAGNET SIMULATION}

The clamping forces for the $\mathrm{CM}$ with the dimensions of the pole edge ( $c \times d=20 \times 40 \mathrm{~mm}$ ) and height $l=5 \mathrm{~mm}$ at various distances $\delta_{0}$ from the plane of the magnet's pole edge and the slope angles $\gamma$ are calculated with the help of the stated technique.

The relief of the density distribution of the vertical $z$ component $f_{z}$ of the force and the horizontal $x$ component $f_{x}$ of the force over the surface of the magnet's pole edge is presented in Fig. 6 in a dimensionless scale (without a $\sigma_{M} J / 4 \pi$ coefficient).

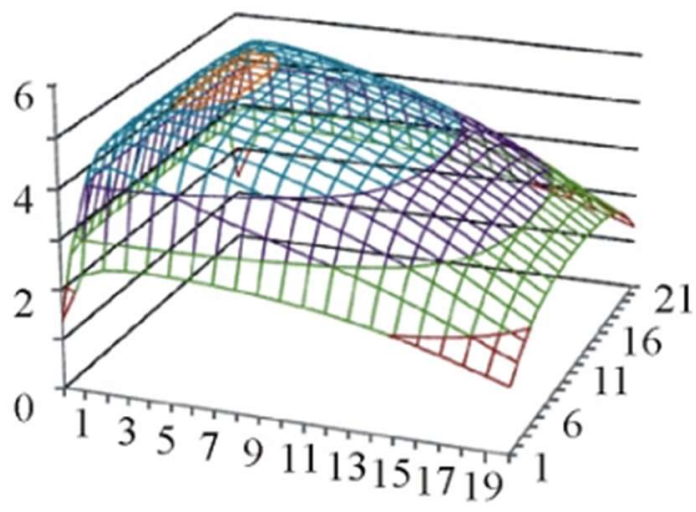

$\mathbf{a}$

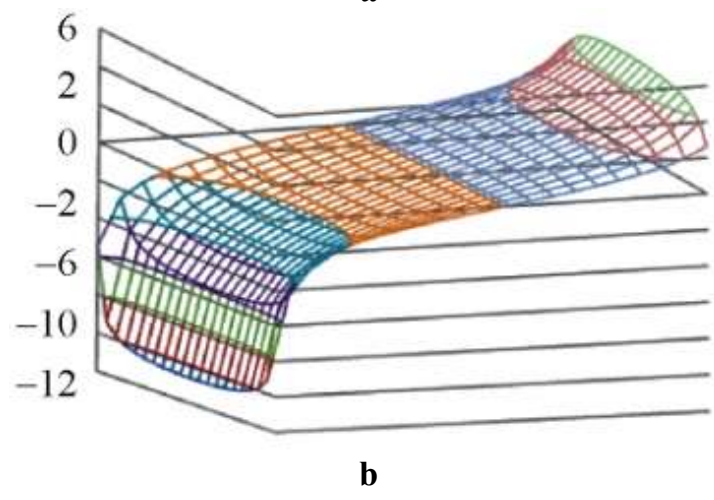

Fig. 6 - Patterns of density distribution for the vertical $f_{z}$ and horizontal $f_{x}$ components of clamping force at $\delta_{0}=2 \mathrm{~mm}$ and $\gamma=10^{\circ}$.

The calculation of the computational points' coordinates and the values of the magnetic field strength components on the basis of the given above formulas is simply carried out using electronic 
computational tables. Moreover, the arrays of the initial and calculated data, presented in a tabular form, make it easy to perform numerical integration operations, vector multiplication and other necessary actions. Implementing the numerical integration of the force density (6) over the area of the pole face, i.e. actually summing each component over all areas, we find the components of the main force vector, that as a whole acts on the pole edge (7).

The components of the main force vector are obtained: $F_{x}=-33.56 \mathrm{H}, F_{z}=210.6 \mathrm{H}-$ for a magnetic system with the mentioned dimensions and a magnet from the $\mathrm{NdFeB}$ material (compound) with a residual induction value $B_{r}=1.0 \mathrm{~T}$ and residual magnetization value $J=800 \mathrm{kA} / \mathrm{m}$, which are typical for such compound, at $\delta_{0}=2 \mathrm{~mm}$ and $\gamma=10^{\circ}$.

The component $F_{y}$ of the resultant vector along the $y$ axis turns out to be zero $\left(F_{y}=0\right)$ plane due to the symmetry of the system and the distribution of the $y$-component of the field and the force density $f_{y}$ relatively the $O x z$ coordinate plane. Then, the absolute value of the resultant vector is

$$
F_{\mathrm{M}}=\sqrt{F_{x}^{2}+F_{y}^{2}+F_{z}^{2}}=213 \mathrm{H} .
$$

To move from the reflected space to the real one, it is necessary to display the virtual concentration center of the forces (point $Q$ ) and transform the coordinate projections of the resultant vector into components, which are naturally formed in the process of interaction of the CM with the FS, namely: the $\mathrm{CF} F_{n}$, directed along the normal to the FS, i.e. perpendicular to the $\xi$ axis; the magnet displacement force $F_{\tau}$, tangential to the FS, i.e. parallel to the $\xi$ axis (see Fig. 4).

Then, using the common formulas for transforming the component vectors when rotating the coordinate axes, we receive:

$$
\begin{gathered}
F_{\tau}=F_{x} \cos \gamma+F_{z} \sin \gamma=3.51 \mathrm{H} ; \\
F_{n}=-F_{x} \sin \gamma+F_{z} \cos \gamma=213 \mathrm{H} .
\end{gathered}
$$

The calculation of the efforts by the stated procedure for a zero gap (or a very small technological gap, that is formed as a result of covering the poles by a material with a large coefficient of friction) gives the same results as Maxwell's well-known equation [27], in which $S_{\mathrm{M}}$ is the area of the pole edge, and in particular, for the considered example

$$
F_{n}=\frac{B_{r}^{2}}{2 \mu_{0}} S_{M}=\frac{1,0^{2}}{2 \cdot 4 \pi \cdot 10^{-7}} 8 \cdot 10^{-4}=318 \mathrm{H} .
$$

At the same time, according to (1) and (7) equations for the gap $\delta=0.01 \mathrm{~mm}$ and $\gamma=0^{\circ}$ we get

$$
\begin{aligned}
& F_{n}=\Delta s_{k}^{\prime}\left(\sum_{k} f_{z}\left(x_{k}^{\prime}, y_{k}^{\prime}\right)\right) \frac{\mu_{0} J^{2}}{4 \pi}=2 \cdot 10^{-6} \times \\
& \times 2510 \frac{4 \pi \cdot 10^{-7}}{4 \pi}\left(800 \cdot 10^{-3}\right)^{2}=321 \mathrm{H} .
\end{aligned}
$$

The obtained results allow to determine all components of the traction efforts and to calculate the required power of the traction drive of the MR's wheel-mover using the fundamental static theorem. In addition, knowing the maximum $\mathrm{CF}$ of a single magnet with a pole area $S_{\mathrm{M}}=8 \mathrm{sm}^{2}$, it is possible to determine the required number of such magnets to keep the total technological weight of the MR on the overturned surface, and also on an inclined surface with a given friction coefficient for FS and various non-ferromagnetic uncertainties.

\subsection{CLAMPING ELECTROMAGNET SIMULATION}

In this paper, for MR's CE numerical simulation it is decided to use the powerful system Ansoft Maxwell 13.0, which is the advanced software for calculation and simulation of electromagnetic fields. This software is used for development and study of two- and three-dimensional models of sensors, motors, transformers and other electric and electromechanical devices for a wide range of applications [26]. Algorithmic basis of Ansoft Maxwell is the finite elements method that accurately calculates the static, harmonic electromagnetic and electric fields. The use of such software significantly reduces the design time, gives the opportunity to optimize the design of $\mathrm{CE}$ in accordance with the basic criteria.

It must be noted that imitation of MR real operating conditions requires extensive consideration. Often MR moves along complex ferromagnetic surface with undefined irregularities: nonferromagnetic gap $\delta$ between $\mathrm{CE}$ and ferromagnetic surface as well as thickness of ferromagnetic surface $s$ (according to Fig. 1) which vary in real conditions. Thus, it is necessary to identify the influence of those parameters $(\delta$ and $s$ ) on the resulting value of clamping force generated by CE. Thickness of ship hulls typically varies from 2 to $50 \mathrm{~mm}$, and thickness of fouling growth varies from 5 to $40 \mathrm{~mm}$.

The authors developed the simulation model that corresponds to the real reduced $\mathrm{CE}$ with an extensive magnetic system $[26,27]$ and the following dimensions: $a=50,2 \mathrm{~mm} ; b=31,0 \mathrm{~mm} ; l$ $=10,0 \mathrm{~mm}(a-$ width,$b-$ length, $l-$ magnet 
height). The spatial position of CE can be specified in the program according to the nature of the working surface (Fig. 7). In particular, the obstacles, placed on the surface, cause different values of the distance from the tops of the magnet [18] to the ferromagnetic surface $\delta_{1}, \delta_{2}, \delta_{3}, \delta_{4}$ (are not shown in Fig. 7). Accordingly, angles of the CE surface inclination to the working surface may also have different values. In addition, the CE's coil parameters are set in the program (number of turns is 1080 and magnetomotive force is $300 \mathrm{~A}$ ). The carbon steel is chosen as magnetic circuit material. Also, electromagnetic processes are modeled in Ansoft Maxwell for the $\mathrm{CE}$ given under the following conditions: 20 maximum number of passes, $0.5 \%$ percent error and for vacuum, which is similar to the air by its magnetic properties, as well as organic substances that cause the surface uncertainty (not ferromagnetic in nature).

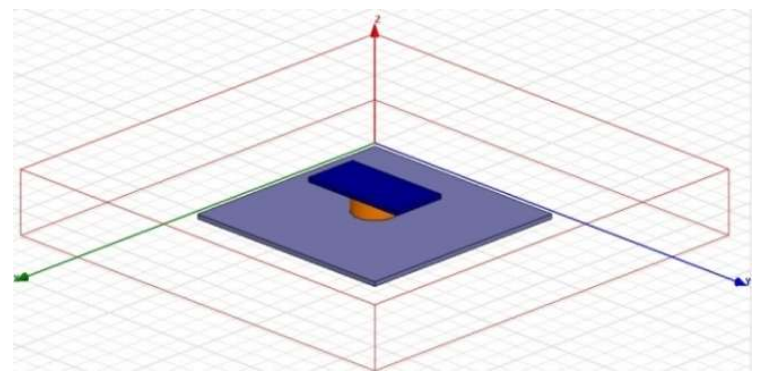

Fig. 7 - Computer simulation model of MR's CE.

The distribution pattern of magnetic induction in the ferromagnetic working surface turned to the $\mathrm{CE}$ of MR is calculated for $4 \mathrm{~mm}$ ferromagnetic plate thickness as well as 1.2 and $1.7 \mathrm{~mm}$ gap (Fig. 8) using a computer model developed by the authors. The values of magnetic induction are obtained in the range of 0.026 to $0.212 \mathrm{~T}$ in the periphery and in the center of the plate, respectively, in front of the magnet for $1.2 \mathrm{~mm}$ gap (Fig. 8, a) and in the range of 0.021 to $0.165 \mathrm{~T}$ in the periphery and in the center of the plate, respectively, in front of the magnet for $1.7 \mathrm{~mm}$ gap (Fig. $8, \mathrm{~b})$. In the first case $(\delta=1.2 \mathrm{~mm}$ ) the calculated value of clamping force is determined by the Maxwell equation [27] and takes $2.16 \mathrm{~N}$, and in the second case $(\delta=1.7 \mathrm{~mm})$ it takes $1.51 \mathrm{~N}$, that is lower value in comparison with the first case.

Also, by restructuring model the distribution of the magnetic field in a working gap (on working ferromagnetic surface) or in any other cross-section of magnetic system "CE-FS" as well as the values of the formed CF can be obtained for CE's static traction characteristics' calculation at different values of magnetomotive force and the gap [27, 28], which is a nonferromagnetic nature uncertainty of working FS and formed due to presence of fouling [26], etc.
At MR's moving on a complex working surface its CE can get to a part of ferromagnetic surface with a smaller thickness due to the surface features or damages. Thus, a ferromagnetic nature uncertainty of working surface is formed. In this case, the phenomenon of the weakening of the magnetic field and, accordingly, the clamping force is observed. Example of the distribution of the magnetic field calculation from the back side of the ferromagnetic plate by changing its thickness and a constant value of the gap is shown in Fig. 9 (with a built finite element grid) [26].

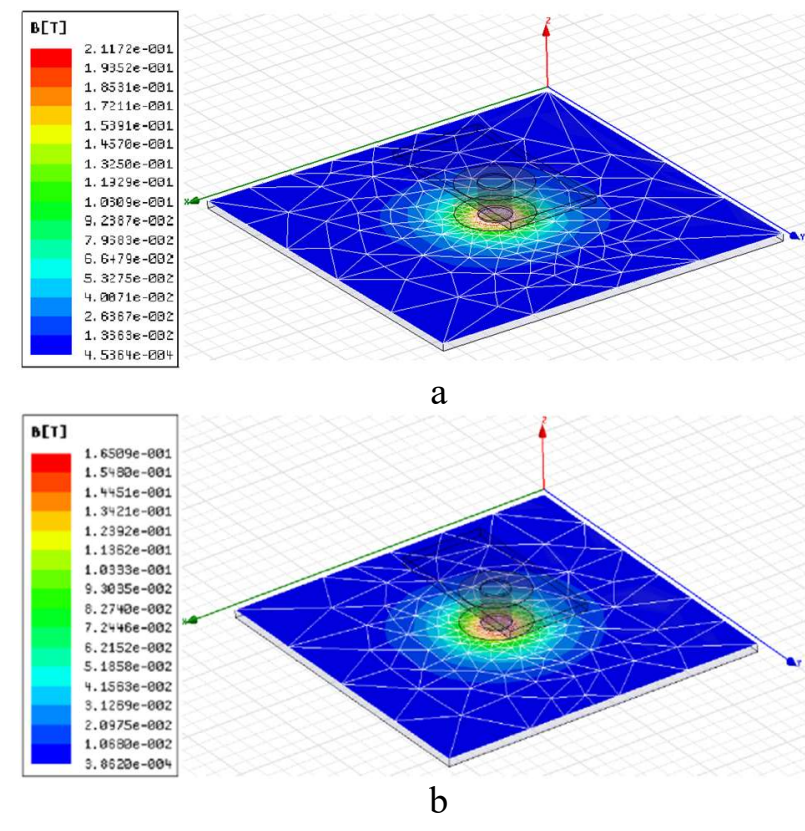

Fig. 8 - Examples of magnetic induction calculation in a ferromagnetic surface $4 \mathbf{~ m m}$ thick in the different values of working gap: $a-1.2 \mathrm{~mm} ; b-1.7 \mathrm{~mm}$.

It should be noted, that there are two ways of solving deterioration problem. The first way is used at small (not enough) partition when accuracy is low, so such solution has a limited value. And the second way is suitable while choosing a large (excess) partition. At first glance a lot of calculation points led to accuracy and validity increasing, but not always a sustainable solution can be obtained in such a case. So, the degree of sampling (the partition limit) of calculation area should be rationally brought out by numerical experiments. And for situation with MR's CE the partition limit was elicited on the basis of the bounds configuration (CE, gap and plate's thickness sizes). So, different spacing between nodes was chosen by the authors in Fig. 9 for $a$ and $b$ cases considering abovementioned notes.

Obtained in Fig. 9 results show the essential weakening of the CE and FS interaction through penetration of the magnetic field outside the plate. Consequently, CE's functioning gets worse at 
clamping force forming and in general when MR moves on complex surfaces. For the greater thickness of the plate $(4 \mathrm{~mm}$ at Fig. $9, \mathrm{~b})$ the lower external field is formed at the plate (the magnetic induction highest value is $2.25 \mathrm{mT}$ at Fig. 9, b) and, respectively, greater magnetic flux is closed through the plate, and higher value of clamping force is formed $(0.96 \mathrm{~N}$ and $1.51 \mathrm{~N}$ in Fig. 9, a and at Fig. 9, b, respectively) [29, 30]. And at plate's thickness reduction the magnetic field that penetrates beyond the plate (the highest value of magnetic induction is $8 \mathrm{mT}$ in Fig. 9, a) increases, respectively, lower magnetic flux is closed through the plate, and a smaller value of clamping force is formed [26].

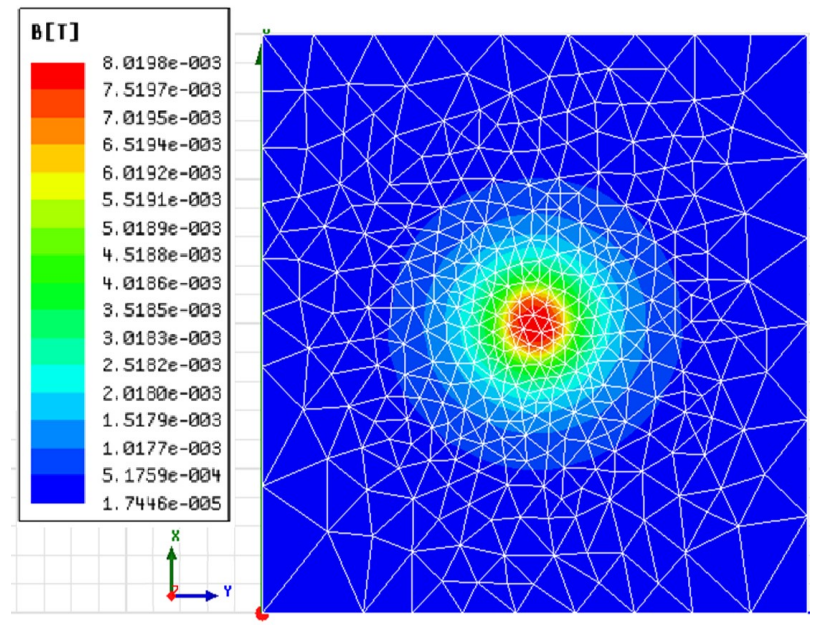

a
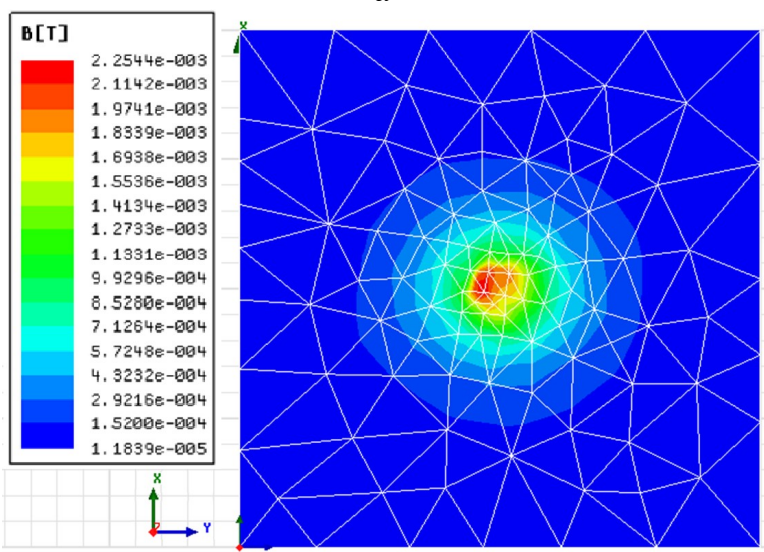

b

Fig. 9 - The patterns of the electromagnetic field penetration beyond the ferromagnetic plate with a non-ferromagnetic gap of $1.7 \mathrm{~mm}$ and different values of its thickness: $a-0.7 \mathrm{~mm} ; \mathrm{b}-4 \mathrm{~mm}$.

Therefore, obtained results show the need to consider not only the thickness of the gap (due to non-ferromagnetic nature uncertainties), but also the thickness of the ferromagnetic surfaces (including the uncertainty of its magnetic properties) to determine the clamping forces and efficiency of the MR's movement at the CE control system designing.

\section{EXPERIMENTAL INVESTIGATIONS OF MR'S ADHESION TO THE WORKING SURFACE}

A series of experimental magnetic induction measurements for different values of the ferromagnetic surfaces' thickness are carried out by the authors using the method of equivalent solenoid $[24,31]$ for modeled CM in Section 5 (see Fig. 7). Fig. 10 shows the schemes for constructing magnetic systems of MR's clamping devices for measuring the values of magnetic induction from the back side of the FS, which simulates the CM and FS interaction in real conditions. Fig. 10, a illustrates the case of a varying gap between the CM and the working FS at its constant thickness (due to the fouling of water organisms - case B in Fig. 1). And Fig. 10, b shows the case of thickness varying of FS with the unchanged gap (due to structural or other features of the surface - the cases E and D in Fig. 1).

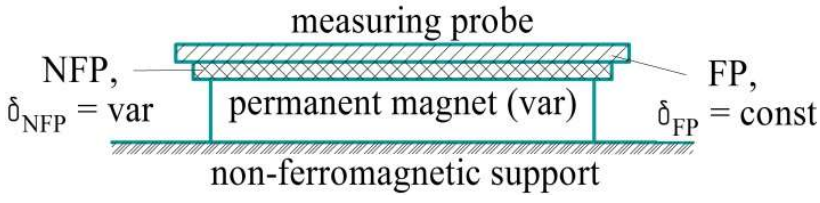

$$
\begin{aligned}
& \text { a } \\
& \text { measuring probe } \\
& \begin{array}{c}
\text { NFP, } \\
\delta_{\mathrm{NFP}}=\mathrm{const}
\end{array} \\
& \text { b }
\end{aligned}
$$

Fig. 10 - Schemes of magnetic induction measuring for various magnets: a - at a constant thickness $\delta_{\mathrm{FP}}$ of the ferromagnetic plate (FP); $b$ - with a varying thickness $\delta_{\text {NFP }}$ of the non-ferromagnetic plate (NFP).

A series of experiments with various permanent and electro-magnets for different thicknesses of the FP and gaps are carried out by the authors. The first thing you need to pay attention to is the accuracy of magnets' manufacturing. In the case of permanent magnets, a different magnetization is observed in the volume of the magnet, which in turn is expressed in different values of the magnetic induction on the outer (clamping) surface of the magnet. This situation can be observed even for magnets from the same batch. Thus, Table 1 shows the results of experiments for two identical neodymium magnets from the same batch with the same nominal declared clamping force of $90 \mathrm{~N}$ with zero gap $\left(\delta_{\mathrm{NFP}}=0\right)$. It can be seen that in some cases, discrepancies can reach more than $60 \%$. Therefore, for the reliable implementation of permanent CM in MR's structures, capable of moving along the FS, it is advisable to equip them with the informationmeasuring system proposed in Section 4. 
The obtained in Section 5 simulation results for $\mathrm{CE}$ are confirmed for an experimental sample. Magnetic induction values measured on the external surface of the different thicknesses (from 0.7 to 4 $\mathrm{mm}$ ) ferromagnetic plates with a constant value (1.7 $\mathrm{mm}$ ) of the gap for different soft-magnetic materials. The penetration of the magnetic field outside of the ferromagnetic plate is from about 3.2 to $12 \mathrm{mT}$, which is of the same order of obtained simulation results (from 2.25 to $8 \mathrm{mT}$ ). Some discrepancy in calculation and experiment is explained by measurement errors of magnet and plate sizes and also magnetic induction values, which are caused by accuracy class of measuring devices. For formed clamping force it is necessary to note the following: at the thickness of the ferromagnetic surface reduction from 4 to $0.7 \mathrm{~mm}$ clamping force loosens from 1.51 to $0.96 \mathrm{~N}$ by calculations and from 3.1 to $2.2 \mathrm{~N}$ by experimental measurements.

Table 1. Results of magnetic induction measurements from the outside of the FP for two permanent CMs.

\begin{tabular}{|c|c|c|}
\hline \multirow{2}{*}{ FP thickness $\left(\delta_{\mathrm{FP}}\right), \mathbf{m m}$} & \multicolumn{2}{|c|}{ Magnetic induction B, mT } \\
\cline { 2 - 3 } & Magnet 1 & Magnet 2 \\
\hline 0 & 250 & 230 \\
\hline 0.5 & 130 & 142 \\
\hline 0.7 & 125 & 136 \\
\hline 0.8 & 125 & 135 \\
\hline 1.1 & 84 & 80 \\
\hline 1.2 & 42 & 47 \\
\hline 1.4 & 32 & 56 \\
\hline 1.5 & 24 & 27 \\
\hline 1.6 & 12 & 15 \\
\hline 2.1 & 2 & 3 \\
\hline 2.4 & 1 & 1 \\
\hline 2.9 & 0 & 0 \\
\hline
\end{tabular}

Therefore, experiment and modeling in general confirms the phenomenon presence of the magnetic field penetration outside the walls of the working surface at the value of its thickness reduction due to damage or structural features that together form ferromagnetic nature surface uncertainties. In practical situations at MR movement on constructively different surfaces the current value of FS thickness $\delta_{\mathrm{FSC}}$ might be less than the predicted value $\delta_{\mathrm{FSP}}$ that is usually taken into account in the design of CEs for MRs. There is a risk of the magnetic field penetration in the other side of the plate with a simultaneous decrease of the magnetic flux in the gap in this case, which brings the clamping force reducing. In fact, this situation is considered unfavorable or even emergency [23] because reduction of the clamping force can lead to undesirable adhesion loss of CE or the whole MR with working surface [26].

Thus, the results indicate that magnetic field distribution and resulting clamping force depend on basic parameters such as: CE's size and magnetomotive force, magnetic permeability of CE's core and material of FS, values of nonferromagnetic gap and FS's thickness. In addition, obtained results can be used in the process of calculation and recovery of the magnetic field on a distributed surface related to the problem of objects identification.

\section{CONCLUSIONS}

The mathematical models of the permanent and electromagnets for the wheeled mobile robots are developed on the basis of the field theory approaches. Obtained equations are solved by: (a) the method of secondary sources for permanent CM using a method of mirror reflections and electronic tables, which consider non-ferromagnetic uncertainties of FS; (b) the method of integrated equations for $\mathrm{CE}$ using finite element method and Maxwell 13 software, which consider ferromagnetic and non-ferromagnetic uncertainties of FS.

The mathematical apparatus of the field theory with computer simulation method allows to design CEs models taking into account the specific features of any CEs constructions and modes, as well as ferromagnetic surface features. Obtained models show basic features of $\mathrm{CE}$ and working surface interaction with quite good accuracy and indicate mentioned interaction as a process with substantially non-linear and undefined parameters. Also it displays the presence of clamping force dependence on the air gap and changes of FS's thickness.

Proposed by the authors control and measuring system of clamping force can be successfully used for new and existing MRs' constructions for their reliability increasing at movement and technological operations performing on complex FSs taking into account the nature of FS's uncertainties (ferromagnetic or non-ferromagnetic).

\section{REFERENCES}

[1] T. Braunl, Embedded Robotics. Mobile Robot Design and Applications with Embedded Systems, Third Edition, Springer-Verlag Berlin Heidelberg, 2003.

[2] V. Gradetskiy, M. Rachkov, Robots for Vertical Movement, Moscow: RF Ministry of Education Publisher, 1997. (in Russian)

[3] Gardit A/S. Environmental Paint Removal with Hydro Blasting / Maritime Surface Protection [Online]. Available: http://goo.gl/EhY99g.

[4] B. Ross, J. Bares, C. Fromme, "A semiautonomous robot for stripping paint from large vessels," Int. J. of Robotics Research, vol. 22, no. 7-8, pp. 617-626, July-August 2008. 
[5] D. Souto, A. Faiña, F. Lopez-Pena, R. J. Duro, "Lappa: a new type of robot for underwater non-magnetic and complex hull cleaning," in Proceedings of the IEEE International Conference on Robotics and Automation (ICRA), Karlsruhe, 6-10 May, 2013, pp. 33943399.

[6] Robot Strips Marine Growth at Sea. JOTUN. Solving the Maintenance Puzzle. Corrosion Protection Systems for Offshore Structures, Subsea Structures, FPSOs. Number 224 [Online]. Available: http://goo.gl/U61X1B.

[7] L. Christensen, N. Fischer, S. Kroffke, J. Lemburg, R. Ahlers, "Cost-effective autonomous robots for ballast water tank inspection," J. of Ship Production and Design, vol. 27, no. 3, pp. 127-136, August 2011.

[8] D. Souto, A. Faiña, A. Deibe, F. Lopez-Peña, R. J. Duro, "A robot for the unsupervised gritblasting of ship hulls," Int. J. of Advanced Robotic Systems, vol. 9, 82, pp. 1-16, 2012.

[9] D. Longo, G. Muscato, "A small low-cost lowweight inspection robot with passive-type locomotion," Integrated Computer-Aided Engineering, vol. 11, pp. 339-348, 2004.

[10] A. N. Tkachenko, N. M. Brovinskaya, Y. P. Kondratenko, "Evolutionary adaptation of control processes in robots operating in nonstationary environments," Mechanism and Machine Theory, vol. 18, no. 4, printed in Great Britain, pp. 275-278, 1983.

[11] S. Osadchy, V. Zozulya, A. Timoshenko, "The dynamic characteristics of the manipulator with parallel kinematic structure based on experimental data," in Proceedings of the IEEE 7th International Conference on Intelligent Data Acquisition and Advanced Computing Systems (IDAACS'2013), Berlin, Germany, September 12-14, 2013, vol. 2, pp. 905-911.

[12] Force Gauge C10 (Maintenance Manual) [Online]. Available: https://goo.gl/2j4s16.

[13] A. Drimus, G. Kootstra, A. Bilberg, D. Kragic, "Design of a flexible tactile sensor for classification of rigid and deformable objects," Robotics and Autonomous Systems, vol. 62, pp. 3-15, 2014.

[14] Y. Kondratenko, O. Gerasin, A. Topalov, "A simulation model for robot's slip displacement sensors," International Journal of Computing, vol. 15, issue 4, pp. 224-236, 2016, http://www.computingonline.net/computing/art icle/viewFile/854/768.

[15] Y. M. Zaporozhets, Y. P. Kondratenko, O. S. Shyshkin, "Mathematical model of slip displacement sensor with registration of transversal constituents of magnetic field of sensing element," Technical Electrodynamics, no. 4, pp. 67-72, 2012. (in Ukrainian)

[16] C. Blanes, M. Mellado, P. Beltran, "Novel additive manufacturing pneumatic actuators and mechanisms for food handling grippers," Actuators, vol. 3, pp. 205-225, 2014. DOI: 10.3390/act3030205.

[17] B.I. Ogorelkov, A.S. Tatevosyan, U.V. Pimonova, D.A. Polyakov, "Experimental research and mathematical modeling of dynamics of the electromagnet of the direct current," Electrical and Data Processing Facilities and Systems, vol. 11, no. 1, pp. 5-14, 2015. (in Russian)

[18] Y.P. Kondratenko, J. Rudolph, O.V. Kozlov, Y.M. Zaporozhets, O.S. Gerasin, "Neuro-fuzzy observers of clamping force for magnetically operated movers of mobile robots," Technical Electrodynamics, no. 5, pp. 53-61, 2017. (in Ukrainian)

[19] L.A. Neyman, V.Yu. Neyman, "Conductivities method application for accounting asymmetrical electromagnet single-side magnetic attraction force," Herald of $\operatorname{IrGTU}$, no. 2 (97), pp. 214-217, 2015. (in Russian)

[20] V.P. Gerdt, A.N. Prokopenya, Simulation of quantum error correction with Mathematica, in V.P. Gerdt, W. Koepf, E.W. Mayr, E.V. Vorozhtsov (Eds.), Computer Algebra in Scientific Computing / CASC'2013, vol. 8136, Lecture Notes in Computer Science, Berlin, Springer-Verlag, pp. 116-129, 2013.

[21] Y.P. Kondratenko, Robotics, automation and information systems: future perspectives and correlation with culture, sport and life science, in: Decision Making and Knowledge Decision Support Systems, Lecture Notes in Economics and Mathematical Systems, vol. 675, Springer, pp. 43-56, 2015. DOI: 10.1007/978-3-31903907-7 6

[22] I.E. Tamm, Foundations of the Theory of Electricity, Moscow: Publisher Nauka, 1976. (in Russian)

[23] M. Pasieka, N. Grzesik, K. Kuźma, "Simulation modeling of fuzzy logic controller for aircraft engines," International Journal of Computing, vol. 16, issue 1, pp. 27-33, 2017, http://computingonline.net/computing/article/vi ew/868.

[24] O.A. Cherkasova, Research of the Magnetic Field of the Permanent Magnet by Means of Computer Modeling. [Online]. Available: https://goo.gl/biytjI. (in Russian)

[25] A.N. Trunov, "An adequacy criterion in evaluating the effectiveness of a model design process," Eastern-European Journal of 
Enterprise Technologies, vol. 1, no. 4 (73), pp. 36-41, 2015. (in Ukrainian)

[26] Y.P. Kondratenko, Y.M. Zaporozhets, J. Rudolph, O.S. Gerasin, A.M. Topalov, O.V.Kozlov, "Features of clamping electromagnets using in wheel mobile robots and modeling of their interaction with ferromagnetic plate," in Proceedings of the 9th IEEE International Conference on Intelligent Data Acquisition and Advanced Computing Systems: Technology and Applications (IDAACS'2017), Bucharest, Romania, 2017, vol. 1, pp. 453-458.

[27] E.T. Markov, Ship Electric Apparatus, 2nd ed., Leningrad: Publisher Shipbuilding, 1981. (in Russian)

[28] S.V. Izmaylov, The Course of Electrodynamics, Textbook for physical and mathematical faculties of teacher training institutes, Moscow: Publisher of Gosudarstvennoe uchebnopedagogicheskoe izdatelstvo ministerstva prosvescheniya RSFSR,1962. (in Russian)

[29] K.M. Polivanov, Theoretical Foundations of Electrical Engineering, Moscow: Publisher Energiya, 1974. (in Russian)

[30] K. Bins, P. Laurenson, Analysis and Calculation of Electric and Magnetic Fields, Trans. from English, Moscow: Publisher Energiya, 1970. (in Russian)

[31] A.N. Tihonov, A.A. Samarskiy, Equations of Mathematical Physics, Textbook for high schools, 5th ed., Moscow: Publisher Nauka, 1977. (in Russian)

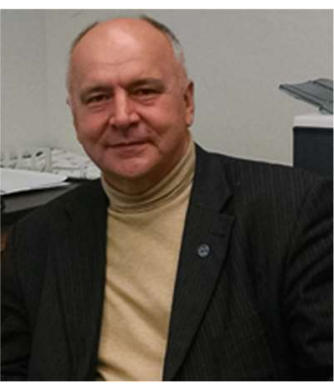

Yuriy Kondratenko, Doctor of Science, Professor, Honour Inventor of Ukraine (2008), Corr. Academician of Royal Academy of Doctors (Barcelona, Spain), Prof. of Intelligent Information Systems at Petro Mohyla Black Sea National University (PMBSNU), Ukraine. He has received (a) the Ph.D. (1983) and Dr.Sc. (1994) in Elements and Devices of Computer and Control Systems from Odessa National Polytechnic University, (b) several international grants and scholarships for conducting research at Institute of Automation of Chongqing University, P.R.China (1988-1989), Ruhr-University Bochum, Germany (2000, 2010), Nazareth College and Cleveland State University, USA (2003), (c) Fulbright Scholarship for researching in USA (2015/2016) at the Dept. of Electrical Engineering and Computer Science in Cleveland State University. Research interests include robotics, automation, sensors and control systems, intelligent decision support systems, fuzzy logic.

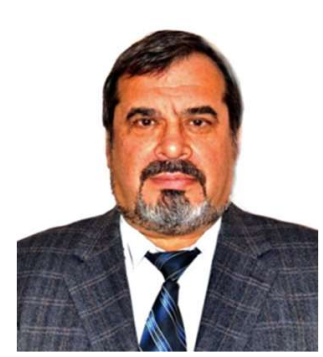

Yuriy Zaporozhets, Superior Civil Servant (2000), Deputy Director of the Shipbuilding Department of the Ministry of Industrial Policy of Ukraine (1998/2006); PhD in theoretical fundamentals of electrical engineering (1983), Professor-consultant at the Department of Intelligent Information Systems of PMBSNU, Associate Professor at the Department of Renewable Energy Sources of National Technical University of Ukraine "KPl" (Kyiv) and the Department of Electrical Machines and Marine Power Systems of Admiral Makarov National University of Shipbuilding (Mykolaiv); Senior Researcher at the Institute of Renewable Energy of National Academy of Science of Ukraine. Awarded the title "Veteran of ships degaussing service". Research interests include energy equipment and systems, energy conversion, automation and processes modeling; characteristics of electromagnetic fields of technical objects; unconventional magnetomechanical interactions.

He has 140 publications, including 16 author's certificates and 5 patents.

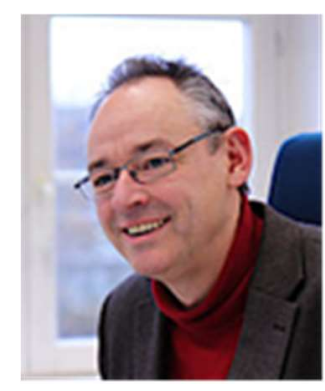

Joachim Rudolph, Prof. Dr.Ing. habil., full professor and head of the Chair of Systems Theory and Control Engineering at Saarland University, Germany. He received diploma (Dipl.-Ing.) in technical cybernetics from Stuttgart University, Germany (1989), doctoral degree from Université Paris Sud, Orsay, France (1991), habilitation in the field of control theory at the Faculty of Electrical and Computer Engineering at TU Dresden, Germany (2003). Memberships: IEEE, IMA, IFAC-TC 2.6, GAMM, VDE, DHV.

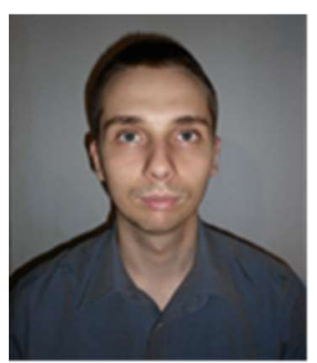

Oleksandr Gerasin, is a lecturer of Admiral Makarov National University of Shipbuilding, Ukraine. $\mathrm{He}$ is specialist in control systems for electric actuators. He has gained several diplomas of bachelor and master of electromechanics.

His research interests include computer control systems, modeling, fuzzy logic, intelligent robotics. 


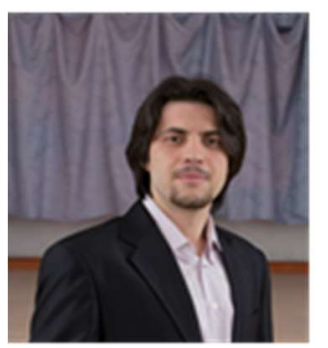

Andriy Topalov, is a lecturer of Admiral Makarov National University of Shipbuilding, Ukraine. He is majoring in electrical engineering. He has gained several diplomas of bachelor and master of electromechanics.

His research interests include computer control systems, sensors, fuzzy sets theory, intelligent robotics devices, and measurement systems.

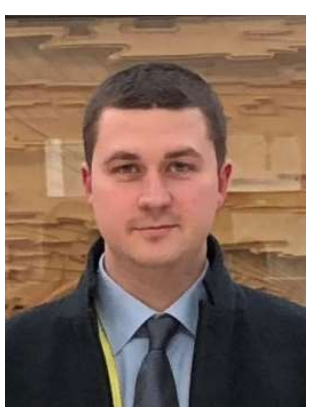

Oleksiy Kozlov, Candidate of Technical Sciences (Ph.D.), Associate Professor of Computerized Control Systems Department of Admiral Makarov National University of Shipbuilding, Ukraine. He is majoring in control processes automation and electrical engineering. He has received the Ph.D. in control processes automation (2014) and master's degree in electromechanics (2011).

His research interests include automation, intelligent control systems, robotics, fuzzy logic, elements and devices of computing systems. 